\title{
A possible mechanism of the protective effect of simvastatin on streptozotocin induced diabetic nephropathy in adult male rats (a histological study)
}

\author{
Original \\ Alshaymaa Gamal Aboulkhair ${ }^{1}$, Marwa sabry ${ }^{1}$ and Ahmed Rabiee ${ }^{2}$ \\ Article \\ ${ }^{1}$ Lecturer of Histology, Department of Histology, Faculty of Medicine, Cairo University, Cairo, \\ Egypt \\ ${ }^{2}$ Lecturer of Internal medicine, Department of Internal medicine, Faculty of Medicine, Cairo \\ University, Cairo, Egypt
}

\begin{abstract}
Background and Objectives: Diabetic nephropathy (DN) is the leading cause of end stage renal disease. The aim of this study is to demonstrate the possible mechanism of reno-protective role of Simvastatin (SMV), a lipophilic compound against streptozotocin induced diabetic nephropathy.

Methods and Results: Thirty-five adult male albino rats were divided into control group, diabetes group and SMV treated diabetic group $40 \mathrm{mg} / \mathrm{Kg}$ / day for 8 weeks. Animals were sacrificed and the kidneys were dissected out and prepared to be stained with hematoxylin and eosin, periodic acid sheif (PAS), toluidine blue and immunohistochemical staining against inducible nitric oxide synthase (iNOS). Ultrathin sections were also prepared. Morphometric measurements and statistical analysis were done. Diabetic group revealed glomerular and tubular affection in addition to significantly increased PAS staining and immunostaining of iNOS. Electron microscopy revealed affected glomerular basement membrane, endothelium and podocyte. Treatment with SMV markedly attenuated these changes with significant decrease in PAS stain and iNOS immunostain.

Conclusion: Simvastatin could attenuate streptozotocin induced DN through many factors as lipo-protection, antiinflammatory and antioxidant effects. This anti-inflammatory and antioxidant effects could be through inhibition of iNOS enzyme and secondary decreased production of NO.
\end{abstract}

Key Words: Diabetic nephropathy, simvastatin (SMV), streptozotocin (STZ), ultrastructure.

Revised: 3 July 2017, Accepted: 8 July 2017

Corresponding Author: Alshaymaa Gamal Aboulkhair, Tel.: 01007385859, E-mail: alshaymaa.aboulkhair@kasralainy. edu.eg

ISSN: 2536-9172, June 2017, Vol.1, No.1

\section{INTRODUCTION}

One of the major complications of diabetes that causes millions of deaths worldwide is DN. Hyperglycemia and advanced glycation end products are risk factors for the development of $\mathrm{DN}^{[1]}$.

Clinically, there is microalbuminuria, which progresses to macroalbuminuria and decreased renal function ${ }^{[2]}$. Histologically, there are glomerular basement membrane thickening, mesangial expansion and tubule-interstitial fibrosis $^{[3]}$.

Diabetic nephropathy is one of the most common causes of end-stage renal disease in many countries leading to an urgent need to find additional effective therapeutic strategies ${ }^{[3]}$. The risk for nephropathy with progression to end-stage renal disease is similar for both types (type I and II) of $\mathrm{DM}^{[2]}$.
Streptozotocin induces diabetes type I by beginning oxidative stress processes that result in the destruction of the Langerhans islets $\beta$ cells. It also inhibits many of the enzymes involved in DNA synthesis and causes degeneration and destruction of DNA (4) Dyslipidemia ${ }^{[5]}$, renal inflammation ${ }^{[6]}$ and oxidative stress ${ }^{[7]}$ have critical role in both initiation and progression of DN.

Oxidativestress increases due to several factorsincluding hyperglycemia, glucose auto oxidation and production of advanced glycation end products that lead to defective antioxidant defenses ${ }^{[8]}$. It increases lipid peroxidation (LPO) and plays an important role in chronic complications of diabetes ${ }^{[9]}$. Additionally, oxidative stress has been known to stimulate inflammatory molecules such as cytokines and inducible Nitric oxide synthase (iNOS) ${ }^{[10]}$.

Inducible Nitric oxide synthase (iNOS) is an enzyme that synthesizes Nitric oxide from L-arginine and molecular 
oxygen. Overproduction of nitric oxide and iNOS has been linked to many chronic inflammatory and immune diseases as a response to bacterial products and pro-inflammatory cytokines $^{[11,12]}$. It is suggested that glomerular iNOS is involved in $\mathrm{DN}^{[13]}$.

Statins (3-Hydroxy-3-methylglutaryl CoA reductase inhibitors) including SMV, block cholesterol biosynthesis and are commonly used to treat dyslipidemia. They could reduce vascular disorders in patients with diabetes ${ }^{[14]}$. They also could be effective in reducing inflammation and glomerular filtration dysfunction in patients with chronic kidney disease ${ }^{[15]}$. Moreover, statins could have an antioxidant activity through improving enzymatic antioxidants; Catalase (CAT), superoxide dismutase (SOD), and glutathione peroxidase (GSH-Px), and nonenzymatic antioxidants; glutathione ${ }^{[16]}$.

\section{AIM OF THE WORK}

This study aims to demonstrate the possible mechanism of reno-protective role of SMV, a lipophilic compound against streptozotocin induced diabetic nephropathy.

\section{MATERIALS AND METHODS}

\section{I-Animals:}

Thirty five adult male albino rats (200- 220 g) were housed in Kasr Al-Ainy animal house, Faculty of Medicine, Cairo University. The animals were chosen after measuring the random blood sugar (RBS) level by glucometer and those rats with RBS less than $200 \mathrm{mg} / \mathrm{dl}$ were chosen. The rats were treated in accordance with guidelines approved by the Animal Use Committee of Cairo University. The rats were provided with ordinary rat chow, bred and housed in wire mesh cages at temperature $24 \pm 1^{\circ} \mathrm{c}$, with normal light-dark cycle. All animals were kept under the same environmental conditions and had free access to water and food.

\section{II-Chemicals used:}

Streptozotocin (STZ), an antibiotic purchased from Sigma Company (St. Louis, MO, USA). It was dissolved in sodium citrate buffer $(0.1 \mathrm{mM}, \mathrm{PH} 4.4)$ immediately before its use.

Simvastatin (ZOCOR) was supplied by Global Napi Pharmaceutical - Egypt under license from: Merk and Co.Inc., Whitehouse Station, N.J., USA in the form of 10 $\mathrm{mg}$ tablets. It was dissolved in distilled water immediately before its use.

\section{III- Experimental design:}

Rats were divided into 3 groups: Group I (Control group. $\mathrm{n}=15$ ): They were subdivided into three subgroups.
Subgroup IA: Five rats received no treatment. Subgroup IB: 5 rats received single intraperitoneal injection of $1 \mathrm{ml}$ of sodium citrate buffer (0.1 mM, PH 4.4) after being starved for 12 hours. Subgroup IC: 5 animals were prepared as in Subgroup IB then 3 days later they received $2 \mathrm{ml}$ distilled water once daily by gastric gavage for 8 weeks. Group II (diabetic group. $\mathrm{n}=10$ ): Rats were injected intraperitoneally with $50 \mathrm{mg} / \mathrm{Kg}$ of STZ after being starved for 12 hours to induce diabetes type $\mathrm{I}^{[17]}$. Streptozotocin was dissolved in sodium citrate buffer $(0.1 \mathrm{mM}, \mathrm{PH} 4.4)$ immediately before its use. After 3 days RBS was measured. Rats with RBS $200 \mathrm{mg} / \mathrm{dl}$ or more were considered diabetic ${ }^{[18]}$. Then the animals were subdivided into: Subgroup IIA (5 rats): received nothing following STZ. Subgroup IIB (5 rats): received $2 \mathrm{ml}$ distilled water once daily by gastric gavage for 8 weeks. Group III (treated group. $n=10$ ): Rats were prepared as in group II then received SMV $40 \mathrm{mg} / \mathrm{Kg}$ dissolved into $10 \mathrm{ml}$ distilled water $(2 \mathrm{ml}$ for each rat) once daily by gastric gavage for 8 weeks ${ }^{[19]}$.

\section{IV-Experimental procedure:}

At the end of the study, RBS, lipid profile, blood Urea and Creatinine were measured in Biochemistry Department, Faculty of Medicine, Cairo University. Blood urea and creatinine were measured using the conventional colorimetric method using Quanti Chrome TM assay kits based on improved Jung and Jaffee methods, respectively (DIur-500 and DICT-500). The animals were sacrificed with intravenous Phenobarbital $(100 \mathrm{mg} / \mathrm{kg})$ and the kidneys were immediately dissected out.

\section{Light microscopic study:}

Specimens from right kidney were fixed in $10 \%$ buffered formalin solution and processed for paraffin sections of 5 micrometers thickness in Histology Department, Faculty of Medicine, Cairo University. Sections mounted on Canada balsam coated slides in case of ordinary and special staining and poly-lysine coated and charged slides in case of immunostaining. Sections of all groups were stained by:

- $\quad H \& E^{[20]}$.

- $\quad$ Periodic Acid Schiff (PAS) stain ${ }^{[21]}$.

- Immunohistochemistry for iNOS antibody ${ }^{[22]}$.

\section{Primary antibody}

It is a rabbit polyclonal antibody (Lab Vision Corporation Laboratories, catalogue number PA121054-).

Detection System for the primary antibody: Ultravision detection system (Lab Vision Corporation Laboratories, catalogue number TP - 015- HD). Reagents Supplied in this kit were; Hydrogen Peroxide Block, Ultra V Block, 
Biotinylated Goat Anti-Polyvalent secondary antibody, Streptavidin Peroxidase, DAB Plus Substrate and DAB Plus Chromogen.

\section{Steps of Immunohistochemical staining:}

Sections were incubated in hydrogen peroxide block for 15 minutes then rinsed in PBS ( 2 times, 2 minutes each). Immunostaining required pretreatment for antigen retrieval, this was done by boiling for 10 minutes in $10 \mathrm{Mm}$ citrate buffer (Lab Vision Corporation Laboratories, cat no AP 9003) pH 6 and leaving the sections to cool in room temperature for 20 minutes then rinsed 4 times, 2 minutes each in Phosphate buffered saline (PBS) (Sigma Chemical CO.P.3813 USA). Sections were incubated immediately with 2 drops of Ultra V Block for 5 minutes at room temperature to eliminate non-specific background. Each section was incubated for 60 minutes with 2 drops $(=100 \mu \mathrm{l})$ of the primary antibody (this step was omitted in negative control sections). Slides were incubated for 10 minutes with 2 drops of Biotinylated Goat Anti-Polyvalent secondary antibody at room temperature then rinsed well with PBS (4 times, 2 min. each). Each section was incubated with 2 drops $(100 \mu \mathrm{l})$ streptavidin peroxidase for 10 minutes at room temperature then washed in PBS (4 times, 2 min. each). One drop (40 ul) DAB Plus Chromogen was mixed with $2 \mathrm{ml}$ of DAB Plus Substrate then applied to section and incubated for 10 minutes at room temperature then rinsed well with distilled water. Slides were counterstained with Mayer's hematoxylin (Lab Vision Corporation Laboratories, cat no TA- 060- MH), dehydrated and mounted. iNOS immunopositive reaction appears as brown cytoplasmic deposits. A photomicrograph of positive control section in lung tissue immunostained with iNOS polyclonal antibody (catalogue number PA121054) was obtained from Lab Vision Corporation Laboratories.

\section{Electron microscopic study ${ }^{[23]:}$}

Small pieces of the left kidney were fixed in $2.5 \%$ glutaraldehyde and postfixed in osmium tetraoxide $1.0 \%$, dehydrated in ascending grades of alcohol, cleared in propylene oxide and embedded in epon. Semithin sections were prepared and stained with toluidine blue. Ultrathin sections were prepared and stained with uranyl acetate and lead citrate and examined by JEOLJEM 1400 transmission electron microscope at electron microscopic unit, Faculty of Agriculture, Cairo University.

\section{Morphometric Study:}

- Optical density of PAS in PAS stained sections

- Area percent of iNOS immunopositive cell
- Basement membrane thickening in EM sections

All measurements were done in 10 non overlapping fields for each animal of each subgroup. Image analysis for light microscopic sections was done using Leica Qwin 500 LTD software image analysis computer system (Cambridge, England) in Histology department, Faculty of Medicine, Cairo University. Image analysis for electron microscopic sections was done using AMTV 600 ADVANTAGE software computer system (Advanced Microscopy Techniques corp. Woburn, United States) at electron microscopic unit, Faculty of Agriculture, Cairo University.

\section{V- Statistical analysis:}

Mean, standard deviation, student's t-test and analysis of variance (ANOVA) were calculated using EXCEL and SPSS 16 software. Results were considered significant when $\mathrm{P}$ value was $<0.05^{[24]}$.

\section{RESULTS}

\section{General Observation:}

No deaths or abnormal behavior were observed in all rats except the presence of excessive urination in the diabetic group. The biochemical, histological and immunehistochemical findings of all control subgroups (group I) were similar. The diabetic subgroups showed also similar biochemical, histological and immune-histochemical findings.

The mean values of random blood sugar level of diabetic rats (group II) were significantly increased when compared to control rats (group I). The mean values of RBS level of the treated rats (group III) revealed nonsignificant decrease eight weeks after SMV intake when compared with diabetic rats (group II) and significant increase when compared with the control rats (group I).

\section{Serum lipid profile levels (Table 2):}

The mean values of low density lipoprotein (LDL), total triglycerides (TG) and total cholesterol (TC) level of diabetic rats (group II) were significantly increased when compared to control rats (group I) while the mean value of high density lipoprotein (HDL) was significantly decreased when compared to control rats. The mean values of LDL, TG and TC level of the treated rats (group III) revealed significant decrease eight weeks after SMV intake when compared with diabetic rats (group II) while the mean value of HDL was significantly increased when compared to diabetic rats. The mean values of LDL, TG, TC and HDL level of the treated rats (group III) revealed nonsignificant change when compared with control rats. 


\section{Urea level (Table 3):}

The mean values of Urea level of diabetic rats (group II) were significantly increased when compared to control rats (group I). The mean values of Urea level of the treated rats (group III) revealed significant decrease eight weeks after SMV intake when compared with diabetic rats (group II). However, the Urea level in group III showed significant increase when compared with the control rats (group I).

\section{Creatinine level (Table 4):}

The mean values of Creatinine level of diabetic rats (group II) were significantly increased when compared to control rats (group I). The mean values of Creatinine level of the treated rats (group III) revealed significant decrease eight weeks after SMV intake when compared with diabetic rats (group II). However, the Creatinine level in group III showed significant increase when compared with the control rats (group I).

\section{II- Histological results:}

\section{Hematoxylin and Eosin stained kidney sections:}

Control group revealed normal structure of the malpighian renal corpuscles, proximal and distal convoluted tubules of the kidney cortex (Fig. 1-a). The diabetic group revealed segmented glomeruli with dilated congested capillaries and markedly dilated Bowman's space. Many tubular and glomerular cells had small darkly stained condensed nuclei. Most proximal convoluted tubules have lost their brush border while other renal tubules showed casts within the lumen. Blood vessels were congested (Fig. 1-b).Sections of treated group III showed a structure nearly similar to that of the control group except for dilated capillaries, mildly dilated Bowman's space and some cells with small darkly stained condensed nuclei. There were partially regained brush border of most proximal convoluted tubules (Fig. 1-c).

\section{Periodic acid Schiff stained kidney sections:}

Control group revealed normal staining of glomerular, capsular, tubular basement membranes, mesangial matrix and brush border (Fig. 2-a).The diabetic group revealed dense staining of the same structures and proximal tubules were seen with many areas of lost brush border (Fig. 2-b). The treated group revealed nearly normal staining of the same structures with few interrupted areas of brush border (Fig. 2-c).

\section{Anti-iNOS stained renal sections:}

Control group revealed negative immunoreactivity in all tubules and glomeruli (Fig. 3-a). The diabetic group revealed strong cytoplasmic and nuclear immunoreactivity in cells of all tubules and glomeruli (Fig. 3-b). Treated group revealed mild cytoplasmic and nuclear immunoreactivity in cells of some tubules and few cells inside some glomeruli (Fig. 3-c).

\section{Semithin renal sections examination:}

Section in the renal cortex of a control rat kidney showed normal glomerular capillaries. PCT cells had vesicular nuclei with distinct brush border and crowded mitochondria (Fig. 4-a). The diabetic group revealed marked dilated congested glomerular capillaries, loss of the brush border of PCT with areas of decreased mitochondria and detached cytoplasm (Fig. 4-b). In treated rats, there was almost restoration of glomerular and tubular architecture except for some dilated congested glomerular capillaries (Fig. 4-c).

\section{Transmission Electron Microscopy of renal sections:}

Control group revealed normal structure of glomerular basement membrane with a central electro-dense layer and bilateral electro-lucent layers. The glomerular basement membrane was surrounded with normal podocyte feet processes on one side and fenestrated endothelium on the other side. Normal mitochondria were seen in both podocyte and endothelial cells (Fig. 5). The diabetic group revealed thick glomerular basement membrane with indistinct layers. Most podocyte feet processes were effaced and fused with each other. The endothelial layer was detached from glomerular basement membrane. Mitochondria were swollen with loss of cristae in both podocyte and endothelial cells (Fig. 6). Sections of treated group showed a structure nearly similar to that of the control group except for small areas with effaced and fused podocyte feet processes (Fig. 7).

\section{III-Morphometric results:}

\section{Optical density of PAS in PAS stained sections (Table 5):}

Optical density of PAS showed significant increase in diabetic group (group II) when compared with control group. Eight weeks after SMV intake, it showed significant decrease in treated rats (group III) when compared with diabetic rats (group II) and nonsignificant increase when compared with control group.

\section{Area percent of iNOS immunopositive cells (Table 6):}

The immunoreactivity was not detected in control rats. It was markedly detected in diabetic rats (group II) and showed significant decrease eight weeks after SMV intake in treated rats (group III). 


\section{Basement Membrane Thickening (BMT) in EM sections (Table 7):}

The mean values of BMT of diabetic rats (group II) were significantly increased when compared to control rats (group I). The mean values of BMT, eight weeks after SMV intake in the treated rats (group III) revealed significant decrease when compared with diabetic rats (group II) and nonsignificant difference when compared with the control rats (group I).

Table 1: The mean values $( \pm \mathrm{SD})$ of random blood sugar level expressed as $\mathrm{mg} / \mathrm{dl}$ of control and experimental subgroups at the end of the experiment.

\begin{tabular}{lcc}
\hline Group & \multicolumn{2}{c}{ Mean $(\mathrm{mg} / \mathrm{dl}) \pm \mathrm{SD}$} \\
\hline I & A & $89.9 \pm 9.4$ \\
& B & $86.3 \pm 10.2$ \\
& C & $91.7 \pm 7.6$ \\
II & A & $309 . \pm 11.9^{* *}$ \\
& B & $307.9 \pm 13.1^{* *}$ \\
III & & $289.8 \pm 10.5^{* *}$ \\
\hline
\end{tabular}

**Significant increase $(\mathrm{P}<0.05)$ as compared to group I

Table 2: The mean values ( \pm SD) of LDL, HDL, TG and TC level expressed as $\mathrm{mmol} / \mathrm{dl}$ of control and experimental groups at the end of the experiment.

\begin{tabular}{lcccc}
\hline Group & $\begin{array}{c}\mathrm{LDL} \\
(\mathrm{mmol} / \mathrm{L})\end{array}$ & $\begin{array}{c}\mathrm{HDL} \\
(\mathrm{mmol} / \mathrm{L})\end{array}$ & $\begin{array}{c}\mathrm{TG} \\
(\mathrm{mmol} / \mathrm{L})\end{array}$ & $\begin{array}{c}\text { TC } \\
\mathrm{mmol} / \mathrm{L})\end{array}$ \\
\hline I & $0.56 \pm 0.05$ & $0.67 \pm 0.05$ & $0.29 \pm 0.01$ & $1.90 \pm 0.07$ \\
II & $1.04 \pm 0.07^{* *}$ & $0.44 \pm 0.02^{*}$ & $0.54 \pm 0.01^{* *}$ & $3.10 \pm 0.17^{* *}$ \\
III & $0.60 \pm 0.08 \square$ & $0.66 \pm 0.05 \square \square$ & $0.30 \pm 0.01 \square$ & $1.87 \pm 0.13 \square$ \\
\hline
\end{tabular}

**Significant increase $(\mathrm{P}<0.05)$ as compared to group I *Significant decrease $(\mathrm{P}<0.05)$ as compared to group I $\square \square$ Significant increase $(\mathrm{P}<0.05)$ as compared to group II $\square$ Significant decrease $(\mathrm{P}<0.05)$ as compared to group II 
Table 3: The mean values $( \pm \mathrm{SD})$ of urea level expressed as $\mathrm{mg} / \mathrm{dl}$ of control and experimental groups at the end of the experiment.

\begin{tabular}{lc}
\hline Group & Mean $(\mathrm{mg} / \mathrm{dl}) \pm \mathrm{SD}$ \\
\hline I & $28.5 \pm 5.1$ \\
II & $87.9 \pm 4.5^{* *}$ \\
III & $52.7 \pm 5.6^{* *} \square$ \\
\hline
\end{tabular}

**Significant increase $(\mathrm{P}<0.05)$ as compared to group I $\square$ Significant decrease $(\mathrm{P}<0.05)$ as compared to group II

Table 4: The mean values $( \pm \mathrm{SD})$ of Creatinine level expressed as $\mathrm{mg} / \mathrm{dl}$ of control and experimental groups at the end of the experiment

\begin{tabular}{lc}
\hline Group & Mean $(\mathrm{mg} / \mathrm{dl}) \pm \mathrm{SD}$ \\
\hline I & $0.62 \pm 0.1$ \\
II & $1.91 \pm 0.087^{* *}$ \\
III & $1.2 \pm 0.081^{* *} \square$ \\
\hline
\end{tabular}

**Significant increase $(\mathrm{P}<0.05)$ as compared to group I $\square$ Significant decrease $(\mathrm{P}<0.05)$ as compared to group II

Table 5: The mean values ( \pm SD) of Optical density of PAS in PAS stained sections of control and experimental groups at the end of the experiment.

\begin{tabular}{lc}
\hline Group & Mean \pm SD \\
\hline I & $0.90 \pm 0.3$ \\
II & $5.9 \pm 0.7 * *$ \\
III & $1.10 \pm 0.4 \square$ \\
\hline
\end{tabular}

**Significant increase $(\mathrm{P}<0.05)$ as compared to group I $\square$ Significant decrease $(\mathrm{P}<0.05)$ as compared to group II

Table 6: The mean values $( \pm$ SD) of area percent of iNOS immunopositive cells in the experimental groups at the end of the experiment.

\begin{tabular}{lc}
\hline Group & Mean Area Percent \pm SD \\
\hline II & $50.9 \pm 7.6$ \\
III & $17.4 \pm 4.8 \square$ \\
\hline
\end{tabular}

$\square$ Significant decrease $(\mathrm{P}<0.05)$ as compared to group II
Table 7: The mean values $( \pm \mathrm{SD})$ of basement membrane thickening level expressed as nanometer (nm) of control and experimental groups at the end of the experiment.

\begin{tabular}{lc}
\hline Group & Mean $(\mathrm{nm}) \pm \mathrm{SD}$ \\
\hline I & $275.8 \pm 34.9$ \\
II & $916.7 \pm 44.3^{* *}$ \\
III & $301.1 \pm 31.5 \square$ \\
\hline
\end{tabular}

**Significant increase $(\mathrm{P}<0.05)$ as compared to group I $\square$ Significant decrease $(\mathrm{P}<0.05)$ as compared to group II

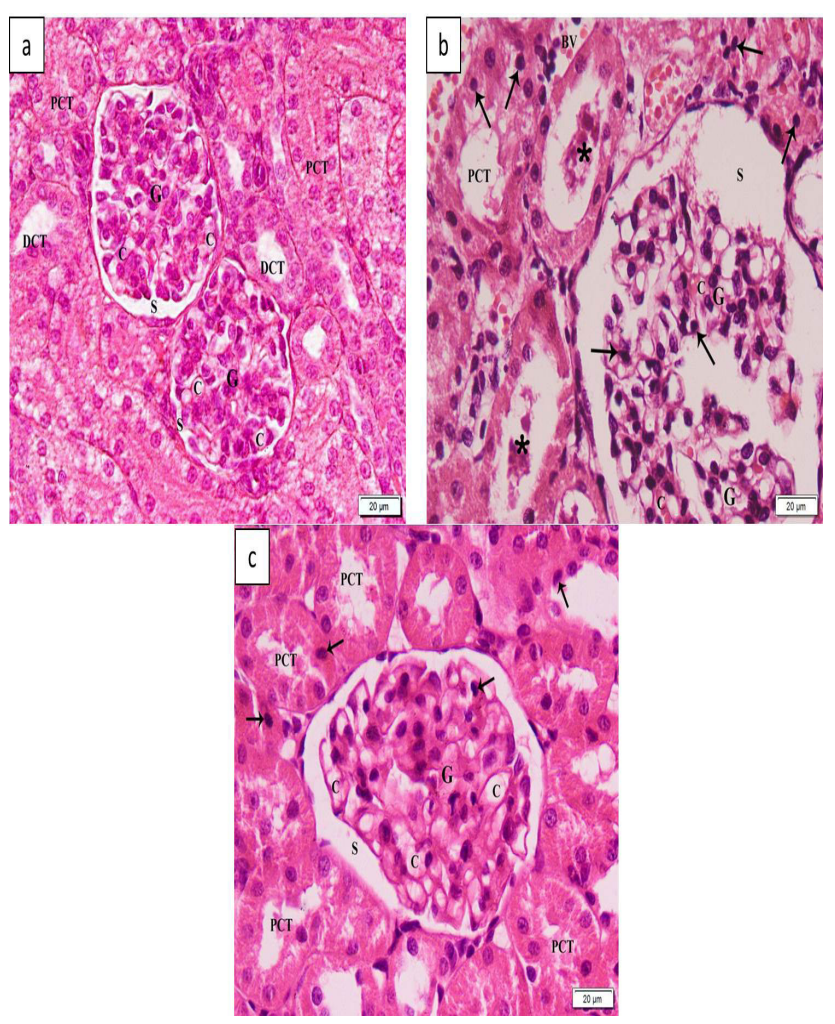

Fig. 1: Photomicrograph of a section in the renal cortex from: (a) a control rat shows two Malpighian renal corpuscles containing glomeruli $(\mathrm{G})$ with many capillaries $(\mathrm{C})$ surrounded with Bowman's space (S), Proximal convoluted tubules (PCT) and Distal convoluted tubules (DCT). (b) a diabetic rat shows segmented glomerulus $(\mathrm{G})$ with dilated congested capillaries (C) and markedly dilated Bowman's space (S). Many tubular and glomerular cells have small darkly stained condensed nuclei (arrows). Proximal tubule is seen with almost lost brush border (PCT). Some renal tubules show casts within the lumen (asterisks). Blood vessels (BV) are seen congested. (c) a treated diabetic rat shows one Malpighian renal corpuscle containing a glomerulus (G) with dilated capillaries (C), mildly dilated Bowman's space (S). Most proximal convoluted tubules (PCT) have partial brush border. Some tubular and glomerular cells have small darkly stained condensed nuclei (arrows). (H\&E X400) 


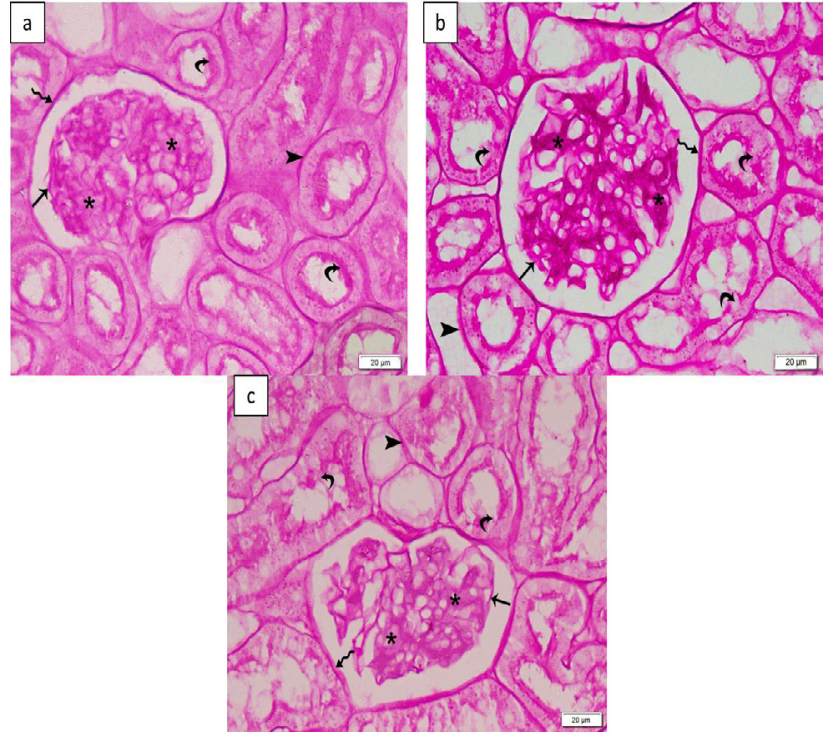

Fig. 2: Photomicrograph of a section in the renal cortex from: (a) a control rat shows normal staining of mesangial matrix (asterisks), glomerular basement membrane (arrow), capsular basement membrane (wavy arrow), tubular basement membrane (arrow head) and brush border (curved arrows). (b) a diabetic rat shows dense staining of mesangial matrix (asterisks), glomerular basement membrane (arrow), capsular basement membrane (wavy arrow), tubular basement membrane (arrow head). Proximal tubules are seen with areas of lost brush border (curved arrows). (c) a treated rat shows almost normal staining of a mesangial matrix (asterisks), glomerular basement membrane (arrow), capsular basement membrane (wavy arrow), tubular basement membrane (arrow head). Brush border shows few interrupted areas (curved arrows). (PAS X400)
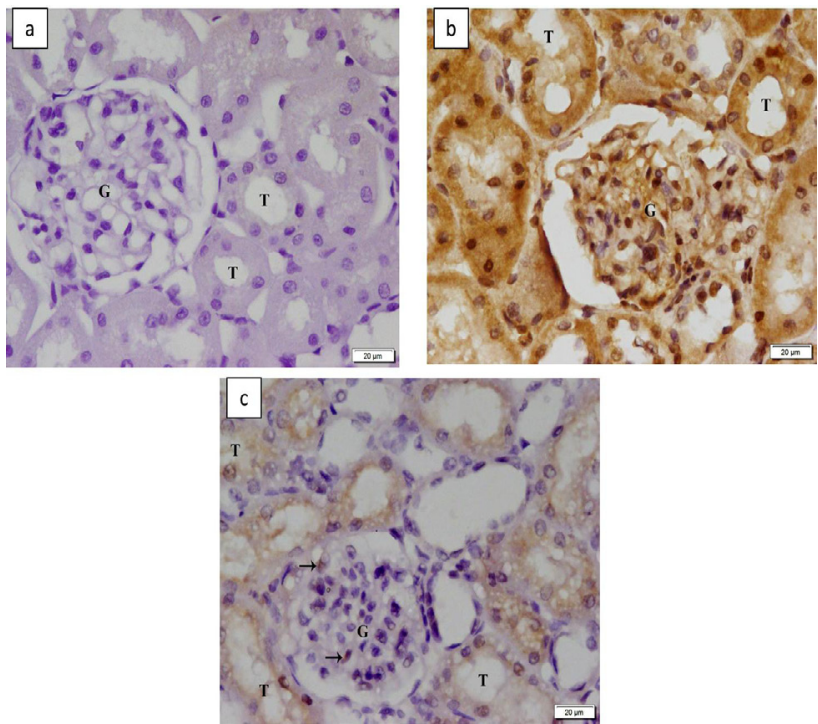

Fig. 3: Photomicrograph of a section in the renal cortex from: (a) a control rat shows negative immunoreactivity in all tubules (T) and glomerulus (G). (b) a diabetic rat shows strong cytoplasmic and nuclear immunoreactivity in cells of all tubules (T) and glomerulus (G). (c) a treated rat shows mild cytoplasmic and nuclear immunoreactivity in cells of some tubules $(\mathrm{T})$ and few cells (arrows) inside glomerulus (G). (iNOS X400)

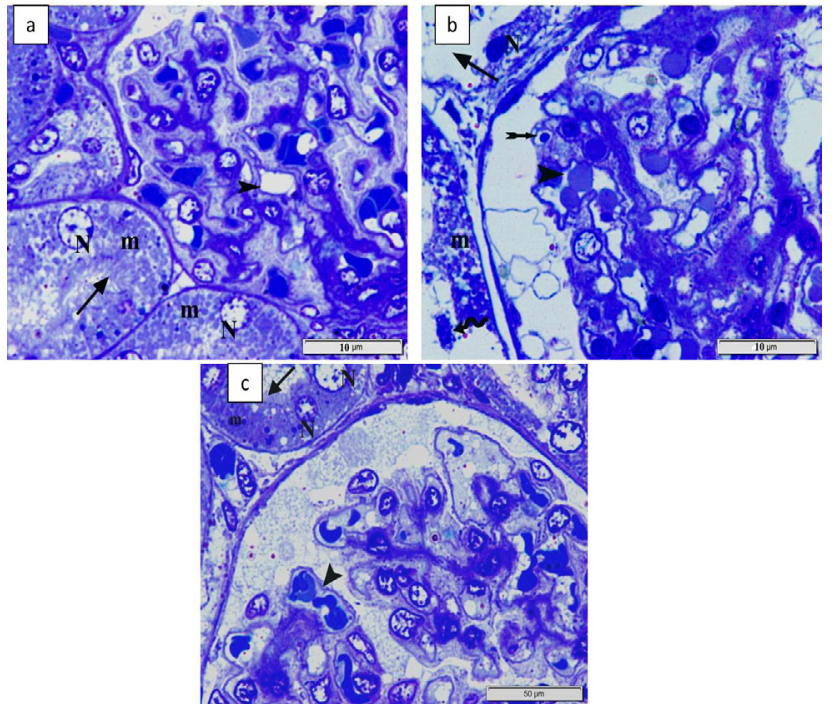

Fig. 4: A photomicrograph of a semithin section in the renal cortex of: (a) a control rat showing normal glomerular capillaries (arrow head). The PCT show vesicular nuclei $(\mathrm{N})$, crowded mitochondria $(\mathrm{m})$ and distinct brush border (arrow). (b) a diabetic rat showing congested dilated glomerular capillaries (arrow head) and small condensed dark nucleus within the glomerulus (arrow with bifid end). The nearside tubules show dark nucleus (N), loss of brush border (arrow) and decreased mitochondria (m). Note the detached cytoplasmic area of a tubular cell (wavy arrow). (c) a treated rat showing congested dilated glomerular capillaries (arrow head). The PCT show vesicular nuclei $(\mathrm{N})$, crowded mitochondria $(\mathrm{m})$ and distinct brush border (arrow). (Toluidine bluex1000)

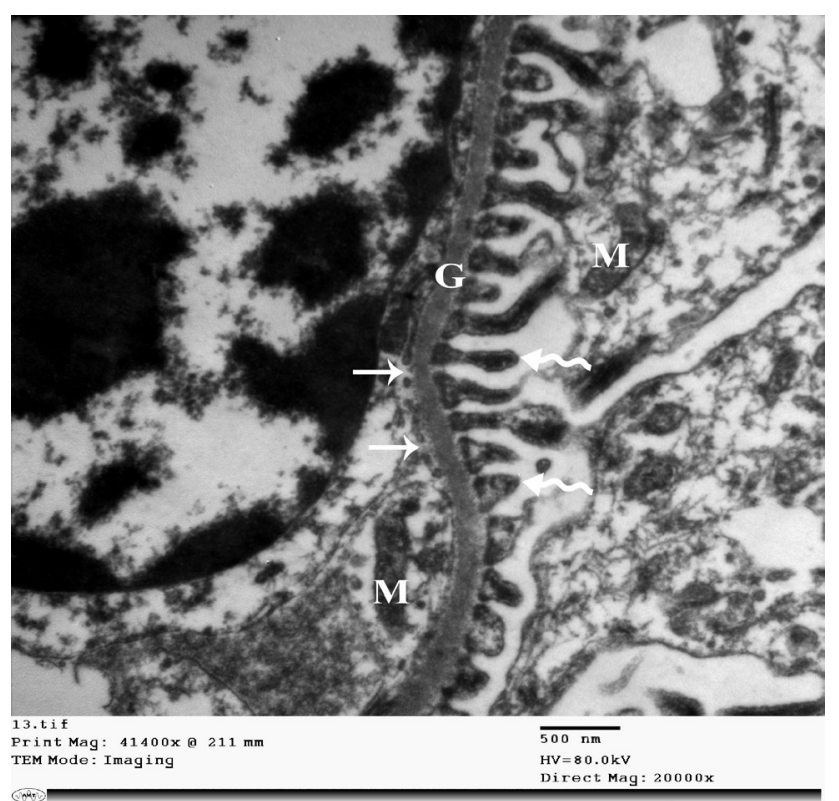

Fig. 5: Photomicrograph of a section in the renal cortex from a control rat shows glomerular basement membrane $(G)$ surrounded with podocyte feet processes (wavy arrows) on one side and fenestrated endothelium (arrows) on the other side. Normal mitochondria $(\mathrm{M})$ are seen in both podocyte and endothelial cells. Note the distinct 3 layers of glomerular basement membrane $(G)$ (central electro-dense layer with bilateral electro-lucent layers). (TEM X20000) 


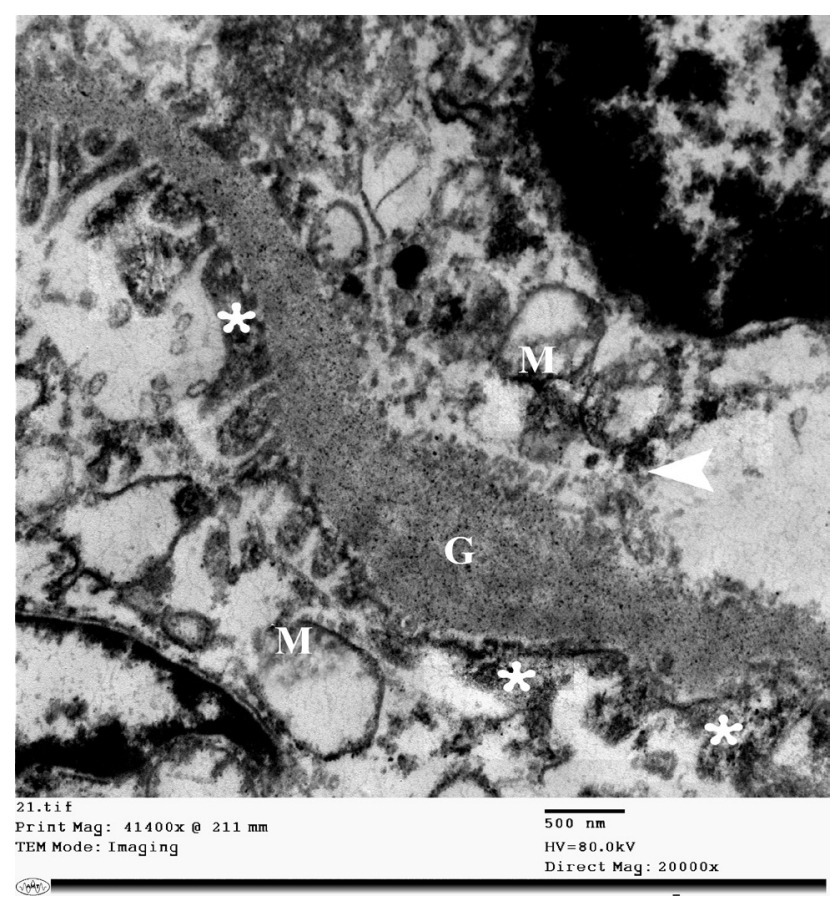

Fig. 6: Photomicrograph of a section in the renal cortex from a diabetic rat shows thick glomerular basement membrane $(G)$ with indistinct layers. Most podocyte feet processes (asterisks) are effaced and fused with each other. The endothelial layer (arrowhead) is seen detached from glomerular basement membrane $(\mathrm{G})$. Mitochondria (M) are swollen with loss of cristae in both podocyte and endothelial cells.(TEM X20000)

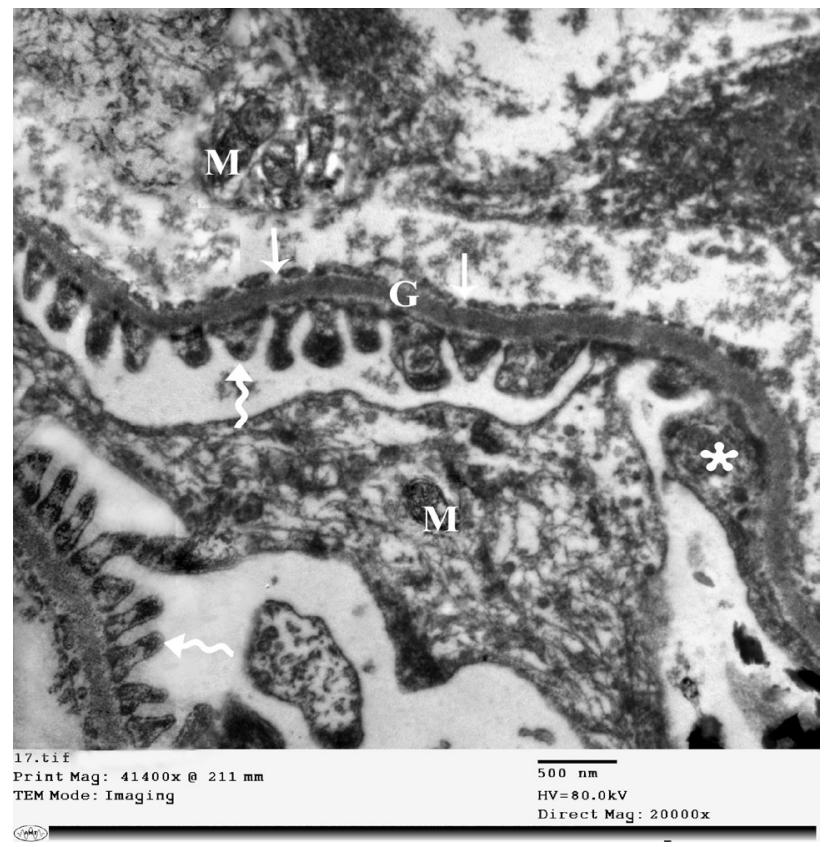

Fig. 7: Photomicrograph of a section in the renal cortex from a treated rat shows glomerular basement membrane (G) surrounded with podocyte feet processes (wavy arrows) on one side and fenestrated endothelium (arrows) on the other side. Normal mitochondria (M) are seen in both podocyte and endothelial cells. A small area with effaced and fused podocyte feet processes (asterisks) is seen. Note the distinct 3 layers of glomerular basement membrane $(\mathrm{G})$ (central electro-dense layer with bilateral electro-lucent layers). (TEM X20000)

\section{DISCUSSION}

In the present study, Three days after STZ administration, diabetes was induced as confirmed by elevated blood sugar level of the rats to $\geq 200 \mathrm{mg} / \mathrm{dl}^{[18]}$. Eight weeks later, DN was confirmed by Serological, histological and ultra-structural measures. Serum urea and creatinine levels were significantly increased in diabetic rats when compared to control rats, indicating renal impairment. This is in agreement with previous studies ${ }^{[16,25]}$ that confirmed the development of $\mathrm{DN}$ by increased creatinine and urea, starting 4 weeks after STZ induction of DM in rats. Serum LDL, TG, TC levels were significantly increased in diabetic rats when compared to control rats while serum HDL was significantly decreased when compared to control rats. This is in accordance with a previous study ${ }^{[26]}$ which stated that diabetic dyslipidemia is characterized by high LDL, TC, TG and low HDL and is considered as an independent risk factor for the progression of diabetic nephropathy.

The histological findings in the current study went parallel with and explained the serological results as they revealed marked kidney injury. The diabetic group in H\&E and semithin sections showed segmented glomeruli, dilated congested glomerular capillaries, dilated Bowman's space and congested blood vessels. Many tubular and glomerular cells had small darkly stained condensed nuclei denoting apoptosis. Most proximal convoluted tubules have lost their brush border while other renal tubules showed casts within the lumen. These findings are concomitant with a previous study ${ }^{[27]}$ which found that examination of renal sections of diabetic rats revealed glomerular atrophy and shrinkage, widening of Bowman's space, atrophy of tubular cells with finally significant loss of renal function. Another study ${ }^{[28]}$ reported that apoptosis was detected in both tubular and glomerular compartments of diabetic kidney and it was obviously shown in podocytes.

These findings could be explained by hyperglycemia that could induce oxidative stress with subsequent tissue injury and inflammation. A previous study ${ }^{[29]}$ reported that hyperglycemia produces reactive oxygen species (ROS) leading to peroxidation of cell membrane lipids, oxidation of proteins and finally renal damage. Moreover, another study ${ }^{[30]}$ stated that oxidative stress is a key process in diabetic complications although the exact mechanisms are partly known.

PAS stained sections from the diabetic group showed dense staining of the glomerular, capsular and tubular basement membranes and mesangial matrix which was confirmed by statistically significant increase in optical density of PAS stained sections of diabetic group when compared with control group. Moreover, the electron microscopic examination revealed thick GBM with indistinct layers. The thickness of GBM was significantly increased in diabetic group when compared with control 
group. These findings could be attributed to increased deposition of basement membrane components and extracellular matrix proteins. These findings are similar to what was detected in a previous study ${ }^{[31]}$ where the GBM thickening was the first change detected after the onset of DM with closely parallel thickening of tubular and capsular basement membranes. Another study ${ }^{[32]}$ stated that prolonged exposure to hyperglycemia caused oxidative stress with further inflammation and production of inflammatory cytokines and growth factors resulting in excessive accumulation of extracellular matrix proteins and renal failure.

Furthermore, the ultrastructral microscopic examination of diabetic group revealed affection of both podocyte and endothelial cells. The podocyte feet processes showed effacement and fusion with each other while endothelial layer was detached from glomerular basement membrane. Mitochondria were swollen with loss of cristae in both cells. These findings are in agreement with a previous study ${ }^{[33]}$ which reported that during development and/or progression of diabetic nephropathy, Podocyte injury is a typical characteristic, togetherwith glomerularbasementmembrane thickening. Moreover, another study ${ }^{[34]}$ reported that injury of both podocyte and endothelial cells together with GBM injury were associated with DN. This study also reported that endothelial cell injury was associated with reduced endothelial glycocalyx secondary to the decrease in glycocalyx proteins including the highly negatively charged versican and decorin. The reduced glycocalyx was associated with decreased fenestrated endothelium area resulting in affection of glomerular filtration barrier together with reduced number of podocyte and flattening of their foot processes.

In the current study, eight weeks after receiving SMV by gastric gavage, treated rats showed partial improvement on the serological, histological and ultra-structural levels. RBS level showed nonsignificant decrease when compared with diabetic group while the mean values of LDL, TG, TC levels of the treated rats revealed significant decrease and the mean value of HDL was significantly increased when compared with diabetic rats.. This is in accordance with a previous study ${ }^{[35]}$ which showed that STZ-induced DN could be attenuated by SMV without having noticeable influence on glycemic control and SMV treatment rescued the dyslipidemia in diabetic rats. Serum urea and creatinine levels showed a significant decrease when compared with diabetic rats, indicating improved renal function. However, their levels showed significant increase when compared with the control rats and couldn't reach the normal level. These findings are in accordance with studies ${ }^{[36]}$ and $^{[16]}$ which stated that administration of SMV tended to significantly reduce serum urea and creatinine and improve kidney function.

H\&E and semithin sections of treated rats showed almost restoration of glomerular and tubular architecture except for dilated capillaries, mildly dilated Bowman,s space and some cells with small darkly stained condensed nuclei. Most proximal convoluted tubules have partially regained their brush borders. PAS staining revealed nearly normal staining of mesangial matrix, glomerular, capsular and tubular basement membranes which was confirmed by significantly decreased optical density of PAS stained sections of treated group when compared with diabetic group. On the ultrastructural level there was a structure nearly similar to that of the control group except for small areas with effaced and fused podocyte feet processes. This was confirmed by measuring the GBM thickness which revealed significant decrease when compared with diabetic rats and nonsignificant difference when compared with the control rats.

These findings are similar to what was detected in a study $^{[35]}$ that revealed partial improvement of histological and ultrastructrul findings in diabetic rats after 13 weeks of SMV intake. They reported improvement of glomerular and tubular injury in H\&E sections together with normal filtration barrier and clearly seen foot processes of podocytes at the ultrastructural level. They reported that, part of the GBM showed increased thickness and considered these pathological changes were slight as compared to diabetic group. Moreover, our results went a line with another study ${ }^{[37]}$ which stated that SMV can reverse podocyte injury in early stage diabetes mellitus through inhibition of vascular endothelial growth factor (VEGF) which is associated with podocyte injury.

These protective effects of SMV on diabetic renal tissues in current study could be explained by the antioxidant activity of SMV which is supported by the study ${ }^{[16]}$ which stated that SMV has a protective effect against STZ-induced oxidative damage by protection against free radicals generation and regulating the enzymatic and non-enzymatic antioxidant systems. Our results may be also explained by the lipophilic protective activity of SMV. Regarding this assumption a previous study $^{[38]}$ reported that statins could reduce renal injury through affecting LDL, high levels of glucose and many other factors mediating the progression of kidney disease.

On the other hand, another study ${ }^{[39]}$ stated that SMV improved VEGF-mediated DN and suggested that the role of statins in DN was independent of their lipidlowering effects. Our findings may be also explained by the anti-inflammatory effect of SMV as a previous study ${ }^{[19]}$ reported that SMV could alleviate diabetes nephropathy by suppressing certain inflammatory signaling molecules as RhoA (Ras homolog gene family, member A) / ROCK1 (rho-associated, coiled-coil-containing protein kinase 1). Inhibition of RhoA/ROCK1 signaling significantly reduced endothelial damage and vascular leakage that was caused by hyperglycemia ${ }^{[40]}$. 
In the current study, immunohistochemical staining of inflammatory mediator iNOS in renal tissue revealed negative immunoreactivity in all tubules and glomeruli of control group. There was strong and mild cytoplasmic and nuclear immunoreactivity in cells of all tubules and glomeruli of diabetic and treated group respectively. The nuclear location of iNOS and other NOS isoforms including neuronal NOS (nNOS) and endothelial NOS (eNOS) have been observed under pathophysiological conditions. Other factors which are important for NO synthesis such as calmodulin and Guanylyl cyclase have also been observed in the nucleus which suggests that nitric oxide could be synthesized in the nucleus and then activate its targets ${ }^{[41]}$.

Other studies ${ }^{[41,42]}$ suggested that, the nuclear translocation of NOS isoforms occurs secondary to various stimuli, and could lead to changes in the regulation of gene transcription, modulation of signal transduction pathways and enzymatic activity with subsequent affection of cells.

The iNOS immunoreactivity was markedly detected in diabetic group. This is in accordance with a study ${ }^{[3]]}$ which reported that the protein expression of iNOS was markedly detected in renal tissues after 8 weeks of induction of DN by streptozotocin in rats. This could be explained by oxidative stress which is considered as one of the factors involved in the development of diabetic complications ${ }^{[44]}$ and it has been recognized as a stimulus for inflammatory mediators, such as cytokines and iNOS (10). Once iNOS is expressed, it produces large amounts of nitric oxide (NO) for prolonged time which acts as an effector molecule in innate immunity and various inflammatory conditions as sustained increased levels of nitric oxide would act as pro apoptotic and stimulator of lipid peroxidation in diabetes ${ }^{[45]}$.

Eight weeks after SMV intake, the area percentage of iNOS was significantly decreased in treated group when compared with diabetic group indicating decreased NO production, inflammation and oxidative stress. This finding is similar to what was detected in a previous study ${ }^{[46]}$ which stated that statins have a range of pleiotropic effects which are independent of its effect on cholesterol metabolism, such as anti-inflammatory and anti-oxidative stress effects. So it is suggested in the current study that the anti-inflammatory and antioxidant effects of SMV in DN may be through inhibition of iNOS enzyme and secondary decreasing production of NO that seems to be a beneficial target to prevent progression of $\mathrm{DN}$.

Contrary to our results, iNOS beneficial effects have been claimed. A previous study ${ }^{[4]}$ mentioned that iNOS may act as anti-fibrotic agent in the vascular system. Another study ${ }^{[48]}$ stated that depletion of tetra- hydrobiopterin, a key co-factor of iNOS activity, appears to be involved in the development of DN in rats. Moreover, a third study ${ }^{[49]}$ suggested that the protective role of statins could be due to the higher availability of NO. So the benefit/damage balance of the observed decrease of iNOS expression by SMV remains to be determined.

\section{CONCLUSION}

Simvastatin could decrease the progression of DN in diabetic rats through its anti-dyslipidemia, antiinflammatory and antioxidant effects as confirmed by improved lipid profile, renal function, histopathology and ultrastructure of renal tissue. Moreover, this study suggests that the anti-inflammatory and antioxidant effects of SMV could be through inhibition of iNOS enzyme and secondary decreased production of NO.

\section{ACKNOWLEDGMENT}

We greatly appreciate our Department's professors for their kind support.

\section{CONFLICT OF INTEREST}

There are no conflicts of interest

\section{REFERENCES}

1. Arora MK and Singh UK: Molecular mechanisms in the pathogenesis of dia $\neg$ betic nephropathy (an update) in Vascul Pharmacol (2013) 58(4):259271.

2. Remuzzi G, Macia $M$ and Ruggenenti P: Prevention and treatment of diabetic renal disease in type 2 diabetes (the BENEDICT study) in J Am Soc Nephrol (2006) 17: 90-97.

3. $\mathrm{Hu}$ YY and Ye SD: Experimental models of type 2 diabetic nephropathy in Chin Med J. (2013) 126(3):574- 7.

4. Akbarzadeh A, Norouzian AD, Mehrabi MR, Jamshidi SH, Farhangi A, Allah VA, Mofidian SMA and Lame RB: Induction of diabetes by streptozotocin in rats in Indian journal of clinical biochemistry (2007) 22 (2): 60- 64 .

5. Kawanami $\mathrm{D}$, Matoba $\mathrm{K}$ and Utsunomiya $\mathrm{K}$ : Dyslipidemia in diabetic nephropathy in Renal Replacement Therapy (2016) 2:16 -24.

6. Navarro-González JF, Mora-Fernández C, De Fuentes MM and García-Pérez J: Inflammatory molecules and pathways in the pathogenesis of diabetic nephropathy in Nature Reviews Nephrology (2011) 7(6): 327-340.

7. Jha JC, Banal C, Chow BS, Cooper ME and Jandeleit-Dahm K: Diabetes and Kidney Disease: 
Role of Oxidative Stress in Antioxidants and Redox Signaling (2016) 25(12): 657 -684.

8. Nowotny K, Jung T, Höhn A, Weber D and Grune T: Advanced glycation end products and oxidative stress in type 2 diabetes mellitus in Biomolecules (2015) 5(1):194 -222.

9. Venturini CD, Merlo S, Souto AA, Fernandes MC, Gomez R, and Rhoden CR: Resveratrol and red wine function as antioxidants in the nervous system without cellular proliferative effects during experimental diabetes in Oxidative Medicine and Cellular Longevity (2010) 3(6): 434-441.

10. Jeong $\mathrm{KH}$, Lee $\mathrm{TW}$, Ihm $\mathrm{CG}$, Lee $\mathrm{SH}$, Moon JY, Lim SJ: Effects of sildenafil on oxidative and inflammatory injuries of the kidney in streptozotocin-induced diabetic rats in Am.J.Nephrol (2009) 29:274-282.

11. Pautz A, Art J, Hahn S, Nowag S, Voss C and Kleinert $\mathrm{H}$ : Regulation of the expression of inducible nitric oxide synthase in Nitric Oxide (2010) 23:75-93.

12. Förstermann $U$ and Sessa WC: Nitric oxide synthases: regulation and function in Eur Heart J (2012) 33 (7): 829-837.

13. Aly M I, Abdalla M N, El Akad M H, El-Sheikh $\mathrm{S}$ and Yousief E M: Role of iNOS and eNOS expression in a group of Egyptian diabetic and non diabetic nephropathy patients in Egyptian Journal of Internal Medicine (2013) 25:33-36.

14. American Diabetes Association: Standards of medical care in diabetes in Diabetes Care (2009) 32(1): 13-61.

15. Wanner $\mathrm{C}$ and Krane V: Sunrise of statins after AURORA and 4D? in $\mathrm{J}$ Am Soc Nephrol (2011) 22(7):11846-

16. Mohamadin AM, Elberry AA, Abdel Gawad HS, Morsy GM and Al-Abbasi FA: Protective Effects of Simvastatin, a Lipid Lowering Agent, against Oxidative Damage in Experimental Diabetic Rats in J Lipids (2011) 2011:167958.

17. Cesaretti, M.L., Ginoza, M., Ribeiro, A.B. and Kohlmann, O: Systemic hemodynamic and left ventricular function of diabetic- induced hypertensive rats in Arq Bras Endocrinol Metabol (2010) 54: 842-851.

18. Bhansali S, Kumar V, Saikia U, Medhi B, Jha V, Bhansali A and Dutta P: Effect of mesenchymal stem cells transplantation on glycaemic profile and their localization in streptozotocin induced diabetic Wistar rats in Indian $\mathrm{J}$ Med Res (2015) 142: 63 -71.

19. Peng H, Luo P, Li Y, Wang C, Liu X, Ye Z, Li C and Lou T: Simvastatin Alleviates Hyperpermeability of Glomerular Endothelial Cells in Early-Stage Diabetic Nephropathy by Inhibition of RhoA/ ROCK1 in PLoS One (2013) 8(11): e80009.

20. Bancroft $\mathbf{J}$ and Gamble M: Theory and Practice of Histological Techniques. $7^{\text {th }}$ ed., staining methods, Churchill Livingstone, Edinburgh, London, Madrid, Melbourne, New York and Tokyo. (2008) pp: 121135

21. Suvarna SK, Layton C, Bancroft JD: Bancroft's theory and practice of histological techniques, 7thedition .Elsevier Health sciences, Churchill Livingstone, (2012) p. 215- 239

22. Bancroft $\mathbf{J}$ and Gamble M: Theory and Practice of Histological Techniques. 7th ed., staining methods, Churchill Livingstone, Edinburgh, London, Madrid, Melbourne, New York and Tokyo. (2008) pp: $263-325$.

23. Suvarna SK, Layton C, Bancroft JD: Bancroft's theory and practice of histological techniques, $7^{\text {th }}$ edition. Elsevier Health sciences, Churchill Livingstone, (2012) p. 493- 538.

24. Emsley R, Dunn G, and White IR: Mediation and moderation of treatment effects in randomised controlled trials of complex interventions in Stat Methods Med Res (2010) 19:237-270.

25. Morsy MA, Ibrahim SA, Amin EF, Kamel MY, Abdelwahab SA, Hassan MK: Carvedilol ameliorates early diabetic nephropathy in Streptozotocin-induced diabetic rats in Biomed Res Int (2014) 2014: 105214.

26. Rosario R and Prabhakar S: Lipids and diabetic nephropathy in Curr Diab Rep (2006) 6: 455- 462.

27. Motshakeri M, Ebrahimi M, Goh YM, Othman $\mathrm{HH}$, Hair-Bejo $\mathrm{M}$ and Mohamed S: Effects of Brown Seaweed (Sargassum polycystum) extracts on kiney, Liver and Pancreas of Type 2 Diabetic Rat Mode in Evidence- Based Complementary and Alternative Medicine (2014) 2014: 379407.

28. Verzola D, Gandolfo MT, Ferrario F, Rastaldi MP, Villaggio B, Gianiorio F, Giannoni $M$, Rimoldi L, Lauria F, Miji M, Deferrari G, Garibotto G: Apoptosis in the kidneys of patients 
with type II diabetic nephropathy in Kidney Int (2007) 72: 1262-1272.

29. Nishikawa T, Kukidome D, Sonoda K, Fujisawa $\mathrm{K}$, Matsuhisa $\mathrm{T}$, Motoshima $\mathrm{H}$, Matsumura $\mathrm{T}$, Araki E: Impact of mitochondrial ROS production on diabetic vascular complications in Diabetes Res Clin Pract (2007) 77(3):41-45.

30. Matough FA, Budin SB, Hamid ZA, Alwahaibi $\mathrm{N}$ and Mohamed J: The Role of Oxidative Stress and Antioxidants in Diabetic Complications in Sultan Qaboos University Medical Journal (2012) 12(1):5- 18.

31. Gonzalez Suarez ML, Thomas D, Barisoni L and Fornoni A: Diabetic nephropathy: is it a time yet for routine kidney biopsy in World $\mathrm{J}$ Diabetes (2013) 4(6):245 -255.

32. Ruggenenti $P$, Carvedi $P$ and Remuzzi G: The RAAS in the pathogenesis and treatment of diabetic nephropathy in Nat Rev Nephrol (2010) 6(6):319 -330.

33. Ilatovskaya DV, Levchenko V, Lowing A, Shuyskiy LS, Palygin $\mathrm{O}$ and Staruschenko A: Podocyte injury in diabetic nephropathy: implications of angiotensin II - dependent activation of TRPC channels in Scientific Reports (2015) 5, ID 17637: 1 -10.

34. Maezawa $\mathrm{Y}$, Takemoto $\mathrm{M}$ and Yokote $\mathrm{K}$ : Cell biology of diabetic nephropathy: Roles of endothelial cells, tubulointerstitial cells and podocytes in J Diabetes Invest (2015) 6: 3-15.

35. Zhang S, Xu H, Yu X, Wang Y, Sun F, Sui D. Simvastatin ameliorates low-dose streptozotocininduced type 2 diabetic nephropathy in an experimental rat model in Int $\mathrm{J}$ Clin Exp Med (2015) 8(4):63886396-.

36. Yao XM, Ye SD, Zai Z, Chen Y, Li XC, Yang GW, Wang YX and Chen K: Simvastatin protects diabetic rats against kidney injury through the suppression of renal matrix metalloproteinase- 9 expression in J Endocrinol Invest (2010) 33(5):292- 296.

37. Wei P, Grimm PR, Settles DC, Balwanz CR, Padanilam PG and Sansom SC: Simvastatin Reverses Podocyte Injury but not Mesangial Expansion in Early Stage Type 2 Diabetes Mellitus in Renal Failure (2009) 31(6) 503- 513.

38. Koya D and Campese VM: Statin use in patients with diabetes and kidney disease.
The Japanese Experience in $\mathrm{J}$ Atheroscl Thromb (2013) 20: 407 -424.

39. Ho C, Hsu YC, Tseng CC, Wang FS, Lin CL and Wang JY: Simvastatin Alleviates DiabetesInduced VEGF-Mediated Nephropathy via the Modulation of Ras Signaling Pathway in Renal Failure (2008) 30:557-565.

40. Mangialardi G, Katare R, Oikawa A, Meloni M, Reni C, Emanueli C and Madeddu P: Diabetes causes bone marrow endothelial barrier dysfunction by activation of the RhoARho-associated kinase signaling pathway in Arterioscler Thromb VascBiol (2013) 33:555-564.

41. Villanueva C and Giulivi C: Subcellular and cellular locations of nitric-oxide synthase isoforms as determinants of health and disease. Free Radic Biol Med. 2010; 49(3): 307-316

42. Schilling K, Opitz N, Wiesenthal A, Oess $\mathrm{S}$, Tikkanen $\mathrm{R}$, Muller-Esterl W, Icking A. Translocation of endothelial nitric-oxide synthase involves a ternary complex with caveolin-1 and NOSTRIN. Mol Biol Cell 2006;17:3870-3880.

43. Hofni A, El-Moselhy MA, Taye A and Khalifa MM: Combination therapy with spironolactone and candesartan protects against streptozotocininduced diabetic nephropathy in rats in Eur $\mathrm{J}$ Pharmacol (2014) 5(744):17382-.

44. Miranda-Díaz A G, Pazarín-Villaseñor L, Yanowsky-Escatell $F$ G and Andrade-Sierra J: Oxidative Stress in Diabetic Nephropathy with Early Chronic Kidney Disease," Journal of Diabetes Research (2016) 2016, ID 7047238: 17 -.

45. Stadler K, Bonini MG, Dallas S, Jiang J, Radi $\mathrm{R}$, Mason RP and Kadiiska MB: Involvement of inducible nitric oxide synthase in hydroxyl radical-mediated lipid peroxidation in streptozotocin-induced diabetes in Free Radic Biol Med (2008) 15: 866-874.

46. Parsaik A.K., Singh B., Hassan M.M., Singh K., Mascarenhas S.S., Williams M.D., Lapid M.I., Richardson J.W., West C.P and Rummans T.A: Statins use and risk of depression: A systematic review and meta-analysis in J. Affect. Disord (2014) 160: 62-67.

47. Gonzalez-Cadavid NF and Rajfer J: Treatment of Peyronie's disease with PDE5 inhibitors: an antifibrotic strategy in Nat Rev Urol(2010 )7(4):215 -21. 
48. Okumura M, Masada M, Yoshida Y, ShintakuH, Hosoi M, Okada N, Konishi Y, MorikawaT, Miura $\mathrm{K}$ and Imanishi $\mathrm{M}$ : Decrease in tetrahydrobiopterin as a possible cause of nephropathy in type 2 diabetic rats in Kidney Int (2006) 70: 471-476.
49. Sabbatini M, Pisani A, Uccello F, Serio V, Serù R, Paternò R, Cianciaruso B, Fuiano $G$ and Andreucci $\mathrm{M}$ : Atorvastatin improves the course of ischemic acute renal failure in aging rats in J. Am. Soc. Nephrol (2004) 15:901 -9. 